\title{
Electric Field and Humidity Trigger Contact Electrification
}

\author{
Yanzhen Zhang, ${ }^{1}$ Thomas Pähtz, ${ }^{2,3, *}$ Yonghong Liu, ${ }^{1, \dagger}$ Xiaolong Wang, ${ }^{1}$ Rui Zhang, ${ }^{1}$ Yang Shen, ${ }^{1}$ \\ Renjie $\mathrm{Ji}^{1}{ }^{1}$ and Baoping Cai ${ }^{1}$ \\ ${ }^{1}$ College of Electromechanical Engineering, China University of Petroleum, 266580 Qingdao, China \\ ${ }^{2}$ Institute of Physical Oceanography, Ocean College, Zhejiang University, 310058 Hangzhou, China \\ ${ }^{3}$ State Key Laboratory of Satellite Ocean Environment Dynamics, Second Institute of Oceanography, \\ 310012 Hangzhou, China
}

(Received 31 March 2014; revised manuscript received 3 July 2014; published 13 January 2015)

\begin{abstract}
Here, we study the old problem of why identical insulators can charge one another on contact. We perform several experiments showing that, if driven by a preexisting electric field, charge is transferred between contacting insulators. This transfer happens because the insulator surfaces adsorb small amounts of water from a humid atmosphere. We believe the electric field then separates positively from negatively charged ions prevailing within the water, which we believe to be hydronium and hydroxide ions, such that at the point of contact, positive ions of one insulator neutralize negative ions of the other one, charging both of them. This mechanism can explain for the first time the observation made four decades ago that wind-blown sand discharges in sparks if and only if a thunderstorm is nearby.
\end{abstract}

DOI: 10.1103/PhysRevX.5.011002

\section{INTRODUCTION}

Contact electrification, which describes the phenomenon that two contacting insulators can acquire electric charges when they are separated, is responsible for numerous mysterious natural phenomena, such as the generation of electrified particles in wind-blown sand and dust [1-4], lightning near volcanic dust plumes [5], and devastating explosions in grain and coal plants [6]. Contact electrification is also extensively exploited in industrial applications, such as electrophotography [7], laser printing [8], 3D printing [9,10], and electrostatic separations [11]. Despite this huge importance of contact electrification for nature and industry, its underlying physics remain elusive [12-16], even though it has been studied since ancient Greece. The main difficulty is to understand how insulators, which by definition have no free charge carriers, can charge one another on contact. In fact, many complex contactelectrification mechanisms, based on electron transfer $[17,18]$, ion transfer [13], transfer of charged material [19], asymmetric partitioning of hydroxide ions [20,21], and nanochemical reactions [22,23], have been proposed, but the scientific debate remains controversial [19,24,25]. For instance, charge transfers between insulators often depend on the contact mode (e.g., point contact, area contact,

*0012136@zju.edu.cn

†Liuyhcup@163.com

Published by the American Physical Society under the terms of the Creative Commons Attribution 3.0 License. Further distribution of this work must maintain attribution to the author(s) and the published article's title, journal citation, and DOI.
Subject Areas: Geophysics

rubbing contact) and other specified conditions [24], with the consequence that certain mechanisms may be predominant for certain conditions but negligible for other conditions.

Moreover, certain phenomena, which are associated with contact electrification, remain insufficiently explained: For instance, in 1971, Kamra made the fascinating observation of electric activity when a thunderstorm blew over gypsum sand dunes in New Mexico [26]. On the top of several dunes, he saw sparks without branches extending from the ground straight up to a few meters high in the air and clearly distinguished them from thunderstorm lightning. However, on days without a nearby thunderstorm but similarly strong winds, Kamra [26] mysteriously did not observe any such sparks. Recently, Ref. [15] also made similar observations in the laboratory: The authors fluidized a particle bed filling a glass jar. With fluidization, the bed particles started to collide with each other. They then acquired large electric charges when a preexisting electric field generated by a van de Graaff generator was applied on the jar but did not do so when the generator was turned off. The relative air humidity in these experiments and Kamra's observation were of comparable magnitude. Reference [15] proposed and simulated a charge-transfer mechanism to explain their laboratory experiments: Under the presence of a sufficiently strong preexisting electric field, oppositely charged charge carriers gather at opposite hemispheres of the insulators before collision, so that at a collision of two insulators, the charge carriers within the colliding hemispheres neutralize each other, which charges both colliding particles. The simulations further considered that particles are neutralized when they hit the grounded bottom, which allowed net charging of the agitated particle cloud, even 
though the charge-transfer mechanism conserves charge. These simulations were in quantitative agreement with the laboratory observations and thus offered a possible explanation for Kamra's [26] observations for the first time [2]. However, since Ref. [15] neither specified the identity of the involved charge carriers nor the manner in which they move within the insulator, a complete picture explaining Kamra's [26] observations has still been missing.

Here, we investigate a contact-electrification mechanism, which is triggered by the interplay of a preexisting electric field $(E)$ and humidity, by experimental means. As we explain in the following sections, we believe this mechanism works in the following way: First, the insulators adsorb water from a humid environment on their surfaces. Then, positively and negatively charged ions dissolved in this water-which we believe to be hydronium and hydroxide ions-polarize and thus gather at opposite sides of the insulators due to the electric field. On contact, they neutralize the respective other species around the contact domain. Finally, when separated, one insulator remains with most of the positively and the other one with most of the negatively charged ions. Apparently, the here-described charge transfer along and between the insulators resembles the aforementioned charge-transfer mechanism of Ref. [15]. However, since the identity of the involved charge carriers and the manner in which they move along the insulator surface are also specified, our study offers a first complete explanation for the aforementioned observations of Kamra [26].

Our study indicates that, at least for point contacts (e.g., interparticle collisions), the mechanism we investigate seems to be much stronger than other contact-electrification mechanisms if the electric field and the relative humidity are sufficiently large. Interestingly, humidity and thus hydronium and hydroxide ions due to the dissociation of water have been associated with a large variety of electrostatic effects and have even been identified as key components in contact electrification in many previous studies [13,20,21,27-32]. Humidity has also been linked to the pickup of insulating particles from a grounded particle bed [33] or from a grounded conductive plane [34] by preexisting electric fields. In fact, in laboratory experiments, such particles were lifted by strong electric fields in the presence of sufficient humidity. It was speculated that these lifts happen because the particles charge via induction (i.e., the redistribution of electrical charge in a conductor due to electric fields) due to water on their surfaces adsorbed from a humid environment. Indeed, the water makes these surfaces conductive, resulting in an upwardly directed lifting force due to the electric field [35]. Note that this speculated lift mechanism strongly resembles our contact-electrification mechanism. The important difference is the contact time: It is not a priori clear that the lifting mechanism, in which particles are in enduring contacts with their conductive surroundings before they are lifted from the surface, can be generalized to a contact-electrification mechanism working for general contacts of insulators, including comparably very brief contacts during interparticle collisions in midair. Moreover, it is actually unclear why adsorbed water on the particle surfaces makes them conductive because experimental studies have shown that water films on particle surfaces may not be continuous but instead covered by a multitude of small water islands, even for very hydrophilic particles [36,37]. In our study, we propose that adsorbed water may make the insulator surfaces quasiconductive even if the water films are discontinuous because charge might be exchanged between these water islands and water vapor surrounding the insulators.

\section{EXPERIMENTS WITHIN SILICONE OIL}

We carry out experiments within cells completely filled with silicone oil. These experiments are described below. (A detailed description of these experiments can be found in Appendixes A and B.) (1) We place a glass bead between two high-voltage (HV) metal electrodes and observe that it bounces back and forth between these electrodes. We measure the absolute value of the charge of the glass bead after contact with these electrodes as a function of $E$ [see Fig. 1(a)]. These experiments confirm that charging of the glass bead occurs during the contact with these electrodes. (2) We place either two or four identical glass beads between these electrodes and observe that they bounce between each other and the electrodes [see Figs. 1(b) and 2 and Supplemental Movie 1 [38]]. In the case of two glass beads, we measure the charges of both beads before and after a collision [see Fig. 1(b)]. These experiments confirm that a large amount of charge is transferred between the two glass beads at contact since the total charge of the two beads remains roughly constant. (3) While in experiments 1 and 2 the glass beads are stored in air before being placed into the silicone oil, we now bake them for several hours to get rid of most of the water on their surfaces. After baking, no bouncing is observed, confirming that the presence of water on the surface of the glass beads is necessary for the their charging. (4) We hang two fibers down a bar. At the end of each fiber, a glass bead is placed, and the distance between the two fibers is exactly one bead diameter, meaning that the glass beads are initially just in contact. If we activate a strong electric field $(E=2.5 \mathrm{kV} / \mathrm{cm})$ directed normal to the contact plain, the glass beads separate [see Fig. 3(b)], while no separation occurs if the electric field is absent [see Fig. 3(a)] or directed parallel to the contact plane [see Fig. 3(c)]. These experiments confirm that a preexisting electric field is responsible for the charging of the glass beads and that the relevant component is the one in the direction normal to the contact plane. Moreover, we now vary the component of $E$ in the direction normal to the contact plane and then, regardless of whether the glass beads separate or not, manually remove one bead, including its fiber, from the setup after turning on the field. After the removal, we increase the electric field to 


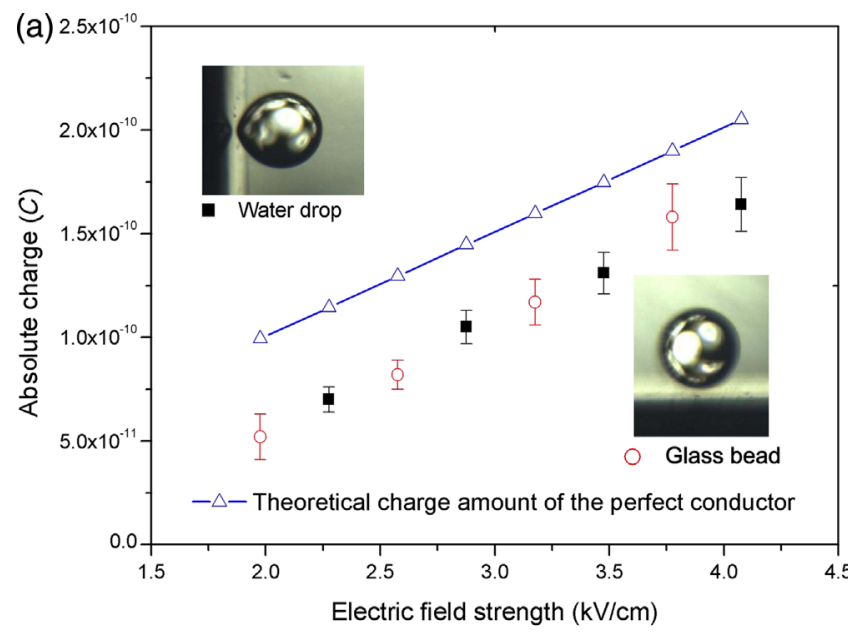

(b)

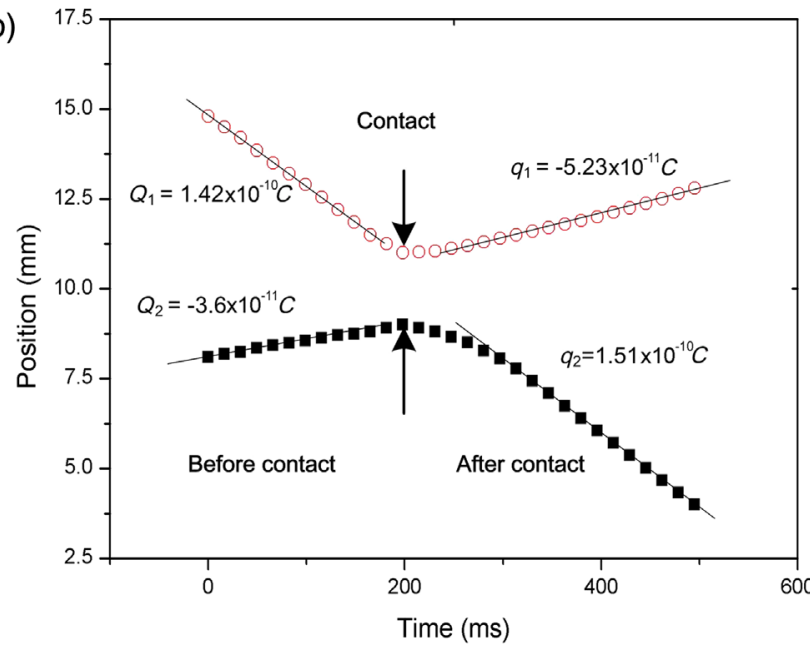

FIG. 1. Experiments within silicone oil: (a) Absolute value of the charges of a glass bead $(r=1 \mathrm{~mm})$ and a water drop $(r=1 \mathrm{~mm})$, bouncing between two high-voltage metal electrodes, after contact with the electrodes versus electric field strength (symbols). The charges are estimated from a balance between the viscous drag and Coulomb forces, while the solid line displays the theoretical charge of a perfectly conducting sphere $(r=1 \mathrm{~mm}$ ) [39]. (b) Positions relative to the cathode and charges of two glass beads colliding between two high-voltage metal electrodes $(E=2.5 \mathrm{kV} / \mathrm{cm})$ before and after their collision $\left(Q_{1}+Q_{2} \approx q_{1}+q_{2}\right)$.
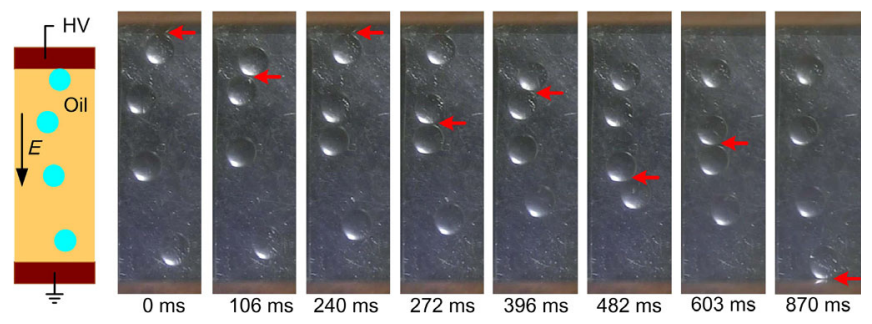

FIG. 2. Experiments within silicone oil: Four glass beads $(r=1 \mathrm{~mm}$ ) bouncing between two high-voltage metal electrodes $(E=2.5 \mathrm{kV} / \mathrm{cm})$. The arrows indicate the locations of charge exchange during each bounce. All pictures show the view from the top of the cell. (a)
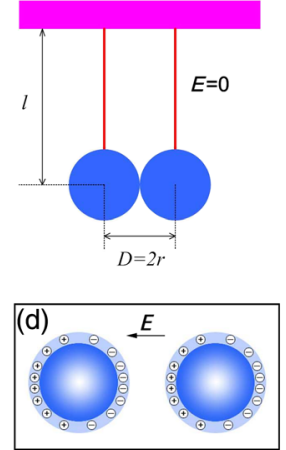

(b)
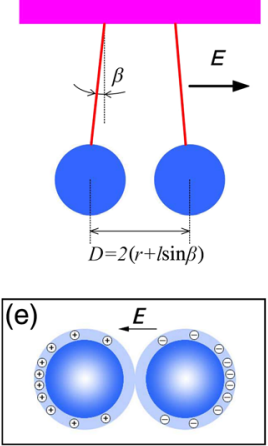

(c)
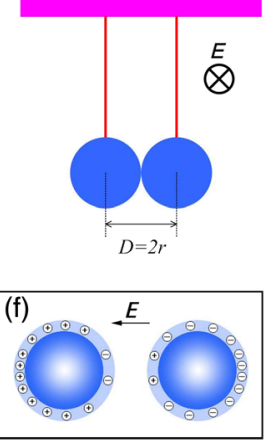

FIG. 3. Experiments within silicone oil: (a)-(c) Two contacting glass beads $(r=1 \mathrm{~mm})$ placed on fibers hanging down a bar. (a) Separation of the glass beads does not occur in the absence of an electric field. (b) Separation does occur under the influence of an electric field $(E=2.5 \mathrm{kV} / \mathrm{cm})$ directed normal to the contact plane. (c) Separation does not occur under the influence of an electric field directed tangential to the contact plane. (d)-(f) Charge-transfer mechanism of hydrophilic particles in contact with each other. Note that the water film is amplified and drawn as continuous for illustration. The real water film is very thin and may not be continuous.

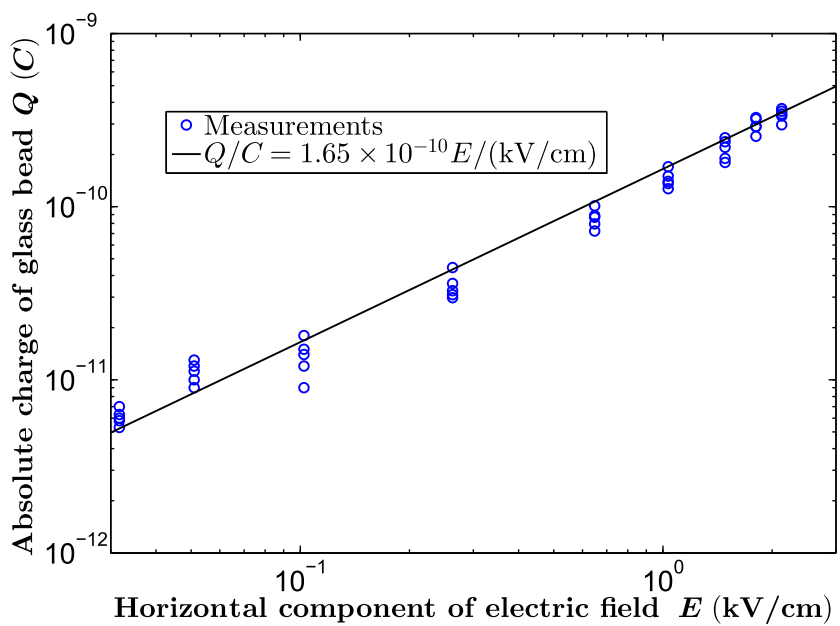

FIG. 4. Experiments within silicone oil: Two contacting glass beads $(r=1 \mathrm{~mm})$ placed on fibers hanging down a bar. Measurements of the absolute value of the charge of the glass beads as a function of the electric field component in the direction normal to the contact plane after removal of one of the beads.

a high value $(E=3.75 \mathrm{kV} / \mathrm{cm})$ and measure the absolute value of the charge of the remaining bead indirectly from its displacement due to the Coulomb force. We find that it is approximately proportional to the electric field component in the direction normal to the contact plane (see Fig. 4).

\section{IDENTITY OF CHARGE CARRIERS}

What are the charge carriers that give the glass beads their charges? The fact that the glass beads must have been 
subjected to a humid atmosphere prior to the experiments to receive charges in contacts indicates that the surfaces of the glass beads have adsorbed water from the atmosphere. (See Appendix D for different adsorption mechanisms.) Hence, the charge carriers are either ions dissolved in the adsorbed water or charge carriers produced by reactions between the insulator surface and substances dissolved within the adsorbed water. (For instance, $\mathrm{O}_{2}$ dissolved in the adsorbed water can considerably increase the surface conductivity of undoped diamond [40].) To minimize the likelihood of chemical reactions with the glass-bead surface, we redo experiments 1 and 2, but this time, we subject the glass beads to a humid $\mathrm{N}_{2}$ atmosphere before placing them into the silicone oil. In fact, we observe exactly the same bouncing behaviors as before. Since $\mathrm{N}_{2}$ is a very inert gas and thus very unlikely to react with the surface of the glass beads, ions dissolved in the adsorbed water remain as the most likely charge-carrier candidates. This hypothesis is further supported by our measurements showing that the absolute charge of the insulator after contact with the electrodes as a function of $E$ resembles that of a water drop [see Fig. 1(a)], indicating that the contact-electrification mechanism is not related to chemical properties of the glass bead. However, one should be aware that this resemblance might be coincidental. Concerning the identity of the ions dissolved in the adsorbed water, we believe, like many previous studies under similar circumstances [13,21,27-30], that the majority of them are hydronium and hydroxide ions due to the reaction $2 \mathrm{H}_{2} \mathrm{O} \rightleftharpoons \mathrm{H}_{3} \mathrm{O}^{+}+\mathrm{OH}^{-}$, especially in the cases in which the glass beads are stored in a humid $\mathrm{N}_{2}$ atmosphere. The reason is that in water saturated with nothing but $\mathrm{N}_{2}$ other ions seem unlikely to be created by standard chemical reactions. Nonetheless, it cannot be excluded that other ions, such as $\mathrm{HCO}_{3}^{-}$due to remnants of $\mathrm{CO}_{2}$ dissolved in the water or ions from substrates with ion bonds contaminating the insulator surface, are significantly or even predominantly involved.

\section{CHARGE-TRANSFER MECHANISM}

Knowing that the charge carriers are most likely ions dissolved in water adsorbed on the insulator surface, it remains to answer the question of what happens to these ions in an electric field before, at, and after contact. Assuming that these charge carriers can be transported along the insulator surface, the electric field, if sufficiently strong, will make the negatively (positively) charged ions gather at the hemisphere closest (farthest) from the anode [see Fig. 3(d)]. At contact, presuming the electric field is normal to the contact plane, the positively charged ions of one glass bead neutralize the negatively charged ions of the other one within a small water bridge forming around the contact point [see Fig. 3(e)]. When the glass beads separate, one glass bead remains with most of the positively and the other one with most of the negatively charged ions [see Fig. 3(f)].
The charge-transfer mechanism described above requires that the ions can be transported along the insulator surface. One possible transport mechanism is that the ions can freely move in a continuous water film coating the insulator surface. However, this possibility seems unlikely since experiments have shown that water films on particle surfaces may not be continuous, even for very hydrophilic particles [36,37]. Instead, particle surfaces might be covered by a multitude of small water islands [36,37]. We thus speculate that the ions instead might hop from water island to water island. This hopping might be possible because each water island might be able to exchange charges with the surrounding water vapor. In fact, charge transfers between metal surfaces and surrounding water vapor under high humidity have been confirmed experimentally [21]. Since the lifetime of adsorbed water molecules is of the order of milliseconds [41], there should always be water vapor surrounding the particles, even in our silicone-oil experiments.

\section{SENSITIVITY OF CHARGE TRANSFER TO SURFACE-MATERIAL PROPERTIES}

Whatever the exact charge-carrier transport mechanism might be, it is at least clear that it is more efficient the more water is available on the insulator surface. This fact suggests that the aforementioned charge-transfer mechanism works better for hydrophilic materials, such as glass, than for hydrophobic materials, such as polyethylene (PE), polystyrene (PS), and polytetrafluoroethene (PTFE). Hydrophilic materials are characterized by large free surface energies, allowing them to form attractive bonds with the water molecules [41]. We indirectly estimate the free surface energy of the insulators by measuring the contact angle between a $1-\mu \mathrm{L}$ water drop and the insulator surface with large contact angles corresponding to small free surface energies. Indeed, we find that the larger the contact angle the more difficult the charge transfer between contacting insulators or between insulators and electrodes (see Fig. 5). We draw this conclusion from quantitative measurements of the absolute charge of spherical beads after contact with the electrodes (see Appendix C) and from qualitative observations of the bouncing behavior of objects (see Supplemental Movie 2 [38]) for different dielectric materials: For instance, bouncing between the electrodes does not occur for the most hydrophobic materials-PE, PS, and PTFE. Also, when PE and PS particles come in contact with the electrodes, they remain in contact and roll on the electrodes for a while before eventually separating. (See Appendix E for a possible mechanism.) PTFE particles, which are more hydrophobic than PE and PS, do not separate at all from the electrodes after contact. In fact, by measuring the average thicknesses of the water covering the surfaces of PTFE and silicon oxide particles (as a representative for hydrophilic materials) as a function of air humidity, we find that the average water thickness on the surface of PTFE particles is between one and two monolayers of water molecules 


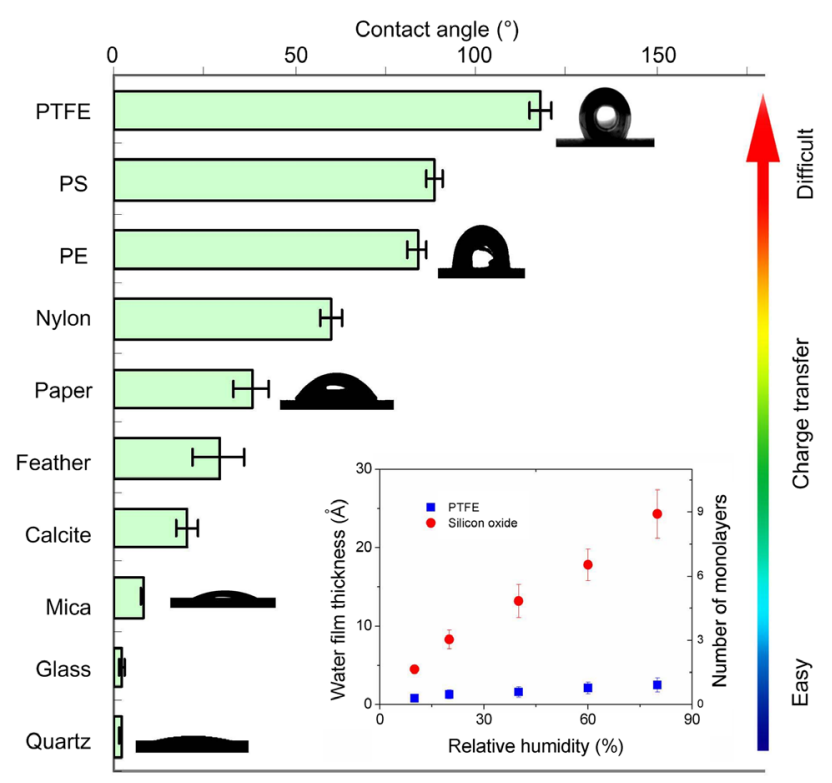

FIG. 5. Difficulty of charge transfer (qualitatively) versus contact angle between a $1-\mu \mathrm{L}$ water drop and the insulator surface for several dielectric materials measured with an optical contact anglemeasuring device. Inset: Number of monolayers of water molecules composing the water films on the surfaces of silicon oxide and PTFE, measured with an ellipsometer, versus air humidity.

almost independent of the air humidity (see the inset of Fig. 5). This thickness is very probably too low to allow efficient ion transport, which explains why contact electrification for PTFE particles is much weaker than for hydrophilic particles. The ability of a PTFE particle to adsorb water can be increased by immersing it in a saturated sodium dodecyl benzene sulphonate ethanol solution. After doing so, the contact angle between the $1-\mu \mathrm{L}$ water drop and the particle surface decreases from about $120^{\circ}$ to about $15^{\circ}$, indicating that a hydrophilic molecular layer with a large free surface energy assembles on the PTFE particle surface. Our measurements show that, after surface modification, the PTFE particles bounce between the electrodes, just like hydrophilic particles (see Supplemental Movie 3 [38]).

\section{CONCLUSION}

What is the relevance of the contact-electrification mechanism we investigate in comparison to previously described mechanisms? To answer this question, we emphasize that the absence of bouncing of glass beads between the electrodes in experiments 1-3 or undetectable glass-bead displacement in experiment 4 do not necessarily imply the absence of contact electrification, for instance, due to previously described mechanisms, because the charging may have been too weak to be noticed in our experimental setups. However, they do imply that the charging is much weaker in comparison to the cases in which bouncing occurs and glass-bead displacement is significant, respectively. Hence, at least for point contacts (e.g., interparticle collisions), the mechanism we investigate seems to be much stronger than other mechanisms if the electric field and the relative humidity are sufficiently large. This particular conclusion is supported by a recent study on contact electrification [42], which experimentally investigated the buildup of electric fields in an agitated particle bed in the absence of a preexisting electric field. This study reported that the agitated bed does not build up an electric field if the relative humidity is too large ( $>45 \%)$. Assuming the correctness of our interpretation of how our contact mechanism works, this observation can be easily explained since our contact-electrification mechanism tends to dissipate existing electric fields. Hence, any attempt of the agitated bed to build up an electric field is countered by the tendency of the field to be dissipated due to our mechanism. This tendency is the stronger the larger the humidity, explaining the existence of a critical humidity beyond which electric fields cannot be built up anymore.

Moreover, it has been shown that the kind of chargetransfer mechanism we describe in Figs. 3(d)-3(f) can lead to a huge buildup of charge in wind-blown particle clouds if the preexisting electric field is sufficiently strong [15]. In fact, even though this charge-transfer mechanism conserves the total charge, a charge buildup can occur due to settling down and neutralization of charged particles at the surface [15]. This charge buildup might explain why Kamra observed sparks at the top of gypsum dunes if and only if a thunderstorm was nearby $[2,26]$ : In fact, the thunderstorm provided a sufficiently strong electric field and humidity (Kamra reported $E \approx 0.04 \mathrm{kV} / \mathrm{cm}$ and $36 \%-58 \%$ humidity) triggering our contact-electrification mechanism. Since, according to our interpretation, this mechanism is based on the charge-transfer mechanism described in Figs. 3(d)-3(f), it leads to the aforementioned huge charge buildup and thus to highly electrified particles, which eventually discharge in sparks.

Finally, the fact that the charging of hydrophilic insulators in electric fields seems to resemble that of water drops [see Fig. 1(a)] might allow controlling contact electrification by controlling the electric fields and the compositions of the insulator surfaces. Exploiting this fact might open new opportunities for industrial applications of contact electrification in the future.

\section{ACKNOWLEDGEMENTS}

We acknowledge support from NSFC Grants No. 51275529 and No. 41350110226 and from the Science and Technology Development Plan of Qingdao City No. 12-1-4-7-(2)-jch. We acknowledge support from Taishan Scholar Construction Project of Shandong Province (Grant No. TS20110823). We thank Fernando Galembeck for discussions.

\section{APPENDIX A: DETAILED DESCRIPTION OF EXPERIMENTS 1, 2, AND 3}

The experimental setup used in experiments 1,2 , and 3 is illustrated in Fig. 6. Two identical copper electrodes are 


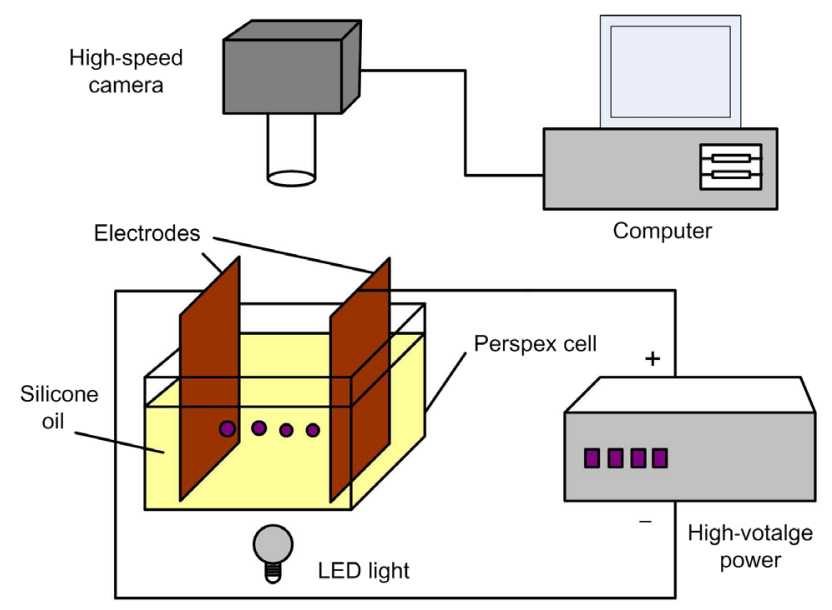

FIG. 6. Illustration of experiments 1,2 , and 3 .

placed in a Perspex cell filled with silicone oil. The width, height, and thickness of the electrodes are 16, 20, and $3 \mathrm{~mm}$, respectively. The distance between the electrodes is $16 \mathrm{~mm}$, and the viscosity $(\mu)$ of the silicone oil is $100 \mathrm{mPa} \mathrm{s}$ at $25^{\circ} \mathrm{C}$. The high-voltage power can be set to values between 0 and $10 \mathrm{kV}$; therefore, the electric field $(E)$ can be set to values between 0 and around $6.25 \mathrm{kV} / \mathrm{cm}$. A highspeed camera is used to record the bouncing movements of the glass beads or the water drops between the electrodes. A light-emitting diode cool light is used in order to avoid heating up the silicone oil. In the water-drop experiments [see Fig. 1(a)], a pipette is used to generate water drops with a radius $(r)$ of $1 \mathrm{~mm}$, which is equal to the radius of our glass beads. All the experimental runs are done at room temperature $\left(25^{\circ} \mathrm{C}\right)$.

An experimental run is started by turning on the electric field and then placing the glass bead(s) or the water drop(s), respectively, one by one, but in fast sequence without interruption, onto the surface of the silicone oil. They sink in, and it takes around 1-2 s time until the glass beads hit the bottom, which is when we start capturing their movement with our camera. During the experimental run, the glass beads slide or roll along the bottom and thereby bounce between the electrodes and each other. In experiments 1 and 2, the glass beads are exposed to air with $50 \%$ relative humidity for two days before the experimental run is started, while they are baked in experiment 3 . The initial horizontal movement of the glass beads in experiments 1 and 2, before they have their first collision with the electrode or another bead, is due to a small amount of excess charge of the adsorbed water [43]. Because of the absence of this charged water in experiment 3 , we initially put the glass beads in contact with the electrodes and/or with each other, but no horizontal movement occurs at all, and all particles settle down at the bottom after a few seconds.

From the images captured by the camera, we determine the velocities of the glass beads or the water drops, respectively. Once obtained, these velocities are further used to determine the charge of the glass beads or the water drops, respectively, as we explain in the following: Since silicone oil is a highly viscous fluid and the velocities of the glass beads are small (the oil is at rest), we can calculate the fluid drag force $\left(\mathbf{F}_{\mathbf{d}}\right)$ on a glass bead using Stokes law

$$
\mathbf{F}_{\mathbf{d}}=-n \pi \mu r \mathbf{U},
$$

where $\mathbf{U}$ is the velocity of the glass bead and $n$ a shape factor that takes into account that the glass bead is not a perfect sphere. ( $n=6$ for a perfect solid sphere.) We determine $n=$ 6.12 from a single glass bead falling with constant settling velocity within the silicone oil. $\left(G-F_{b}+n \pi \mu r U_{z}=0\right.$, where $G$ is the magnitude of the gravitational force on the glass bead, $F_{b}$ the magnitude of the buoyancy force on the glass bead, and $z$ the vertical direction.) It has been shown that Eq. (A1) is also fulfilled for water drops, even though they are liquid, with a coefficient of $n \approx 4[44,45]$. Indeed, from a single water drop falling with constant settling velocity within the silicone oil, we determine $n=3.91$. Knowing $n$, we determine the charge $(Q)$ of the glass bead or the water drop, respectively, within our electric field $(E)$ from the electrodes through $Q E-n \pi \mu r U_{x}=0$, where $x$ is the horizontal coordinate, as

$$
Q=\frac{n \pi \mu r U_{x}}{E},
$$

whereby we neglect the frictional force from the sliding or rolling motion on the bottom because it is typically much smaller than the drag force. We indirectly confirmed this fact by performing an experimental test run in which the glass beads bounce vertically and thus without wall friction instead of horizontally. In this test run, we measure virtually the same charges.

\section{APPENDIX B: DETAILED DESCRIPTION OF EXPERIMENT 4}

The experimental setup used in experiment 4 is illustrated in Fig. 7. Two identical glass beads $(r=2 \mathrm{~mm})$ are hung down a bar using fibers with $10-\mu \mathrm{m}$ diameter and 55-mm length. The glass beads are initially just in contact and put between two identical copper electrodes within a Perspex cell filled with silicone oil. The width, height, and thickness of the electrodes are 20,25, and $3 \mathrm{~mm}$, respectively. The distance between the electrodes is $32 \mathrm{~mm}$, such that the electric field could be set to values between 0 and about $4 \mathrm{KV} / \mathrm{cm}$. A camera is used to record the position of the glass beads during the experiments. The glass beads are exposed to air with $50 \%$ relative humidity for two days before the experimental run is started. All the experimental runs are done at room temperature $\left(25^{\circ} \mathrm{C}\right)$.

An experimental run is started by turning on the electric field. If the electric field component in the 


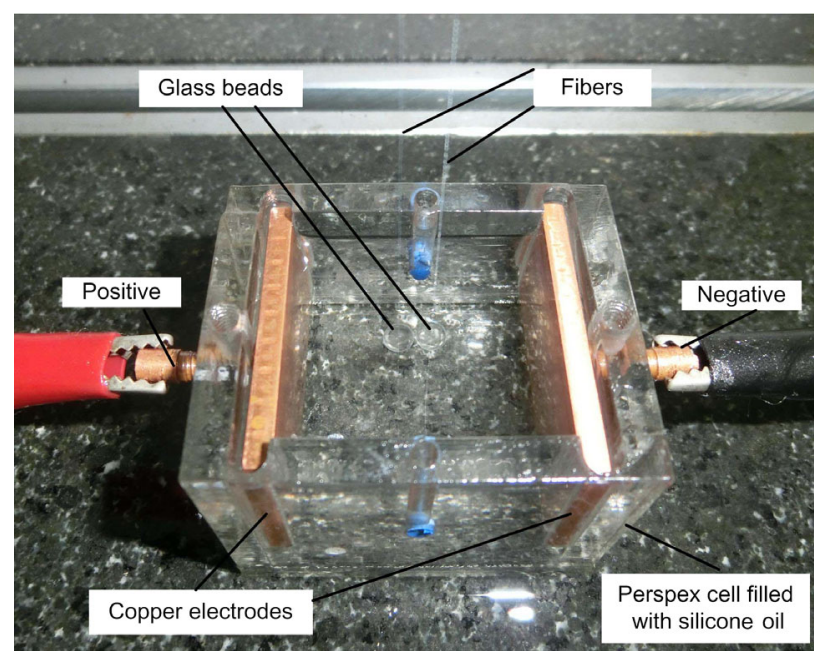

FIG. 7. Illustration of experiment 4.

direction normal to the contact plane $(E)$ is stronger than about $2 \mathrm{kV} / \mathrm{cm}$, the two glass beads separate from each other; otherwise, they do not separate [see Figs. 3(a)-3(c)] because the attractive force (cohesion and/or adhesion) between the two beads is too strong. Still, even if they do not separate, they acquire electric charge, whose value we determine as a function of $E$ (see Fig. 4) in the following way: Regardless of whether the glass beads separate or not, we manually remove one bead, including its fiber, from the setup after turning on the field. Afterward, we increase $E$ to a high value $(3.75 \mathrm{kV} / \mathrm{cm})$ and measure the displacement $(d)$ of the bead due to the Coulomb force by comparing the camera pictures with and without electric field. From $d$, we obtain the charge indirectly from the force balance

$$
Q E=F_{c}=\left(G-F_{b}\right) \tan (\theta)
$$

(see Fig. 8) as

(a)

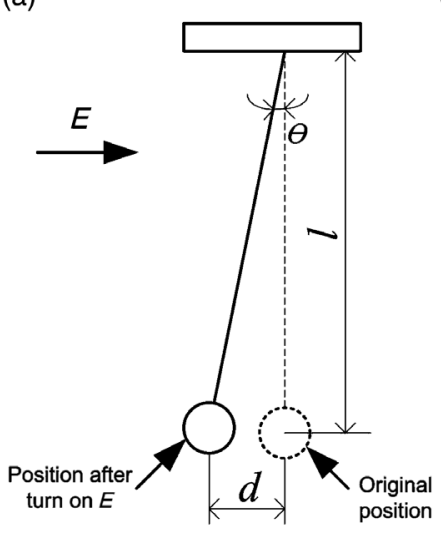

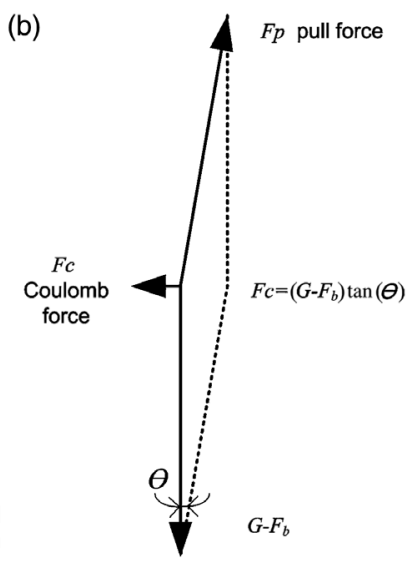

FIG. 8. Sketch of glass-bead displacement due to electric field.

$$
Q=\frac{\left(G-F_{b}\right) \tan \arcsin (d / l)}{E}
$$

We note that, if the electric field is weaker than $0.2 \mathrm{kV} / \mathrm{cm}$, we use longer fibers $(215 \mathrm{~mm})$ in order to better detect the displacement of the glass bead. Furthermore, we wish to emphasize that we verify using the same method that the glass beads do not charge if they are not initially in contact with each other.

\section{APPENDIX C: EXPERIMENTS BEHIND FIGURE 5}

Figure 5 and its insets contain three types of information. First, it shows the contact angle between a $1-\mu 1$ water drop and the insulator surface for different dielectric materials measured with an optical contact angle-measuring device. Second, it shows the average thickness of the water film for silicon oxide and PTFE as a function of air humidity measured with an ellipsometer. Third, it shows the difficulty of charge transfer for different dielectric materials obtained from qualitative observations of the bouncing behavior of objects of different dielectric materials using the experimental setup of experiments 1,2, and 3 described above (see Supplemental Movie 2 [38]). These qualitative observations are further backed up by quantitative measurements of the charge of spherical glass, nylon, PE, PS, and PTFE beads after contact with the electrodes $(E=2.5 \mathrm{kV} / \mathrm{cm})$. In fact, Fig. 9 shows the portion of the charge

$$
Q_{\text {theory }}=\frac{\pi^{2}}{6} 4 \pi r^{2} \epsilon_{o} \epsilon_{r} E
$$

an ideally conducting sphere with radius $r$ would obtain after contact with the electrodes, where $\epsilon_{o}$ is the permittivity of vacuum and $\epsilon_{r}$ the relative permittivity of silicone oil. It can be seen that the more hydrophilic the material

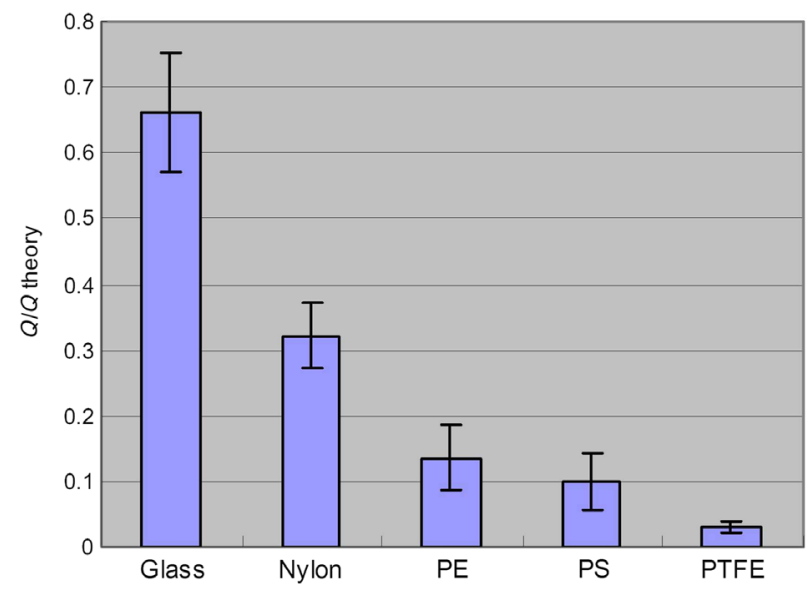

FIG. 9. Charge of spherical beads of different dielectric materials after contact with the electrode. 
the larger the charge after contact with the electrodes (see Fig. 5).

\section{APPENDIX D: WATER ADSORPTION FROM A HUMID ATMOSPHERE}

The amount of water adsorbed by an insulator from a humid atmosphere depends not only on the degree of humidity but also on the chemical properties of the insulator surface. For instance, ionic insulators, such as $\mathrm{NaCl}$ and mica, adsorb water due to electrostatic interactions between the electric fields generated by the ions of the insulator and the dipole and higher moments of the water molecule [46], while hydrophilic covalent insulators, such as glass and quartz, adsorb water due to the generation of strong hydronium bonds [47]. Hydrophobic insulators, such as PE, PS, and PTFE, which do not adsorb water by either of these mechanisms, can still adsorb water due to ubiquitous dispersion interactions [47], however, to a much smaller extent than with the other two mechanisms.

\section{APPENDIX E: POSSIBLE MECHANISM FOR CONTACT ELECTRIFICATION OF HYDROPHOBIC INSULATORS}

Even though contact electrification is strongly resisted by the hydrophobic insulators made of PE and PS, it still occurs. Here, we hypothesize how the charge-transfer mechanism might have looked like in the case of an insulator-electrode contact. First, we note that the size of the water islands on the surfaces of hydrophobic insulators is much smaller than on hydrophilic insulators, and much less water vapor is located near the insulator surface. These issues make charge transport from water island to water island via the aforementioned exchange with surrounding water vapor much more difficult for hydrophobic particles than for hydrophilic particles. We illustrate this difficulty in Fig. 10 through drawing isolated water islands, while the water film on hydrophilic particles is drawn as continuous in Figs. 3(d)-3(f) to illustrate that charge transport along it is easily possible. These water islands, however, still contain small amounts of excess charge [48] and are either positively or negatively charged. [For illustration purposes, the charges are only positive in Fig. 10(a).] On contact with the cathode, the water island at the contact point becomes equipotential with it [see Fig. 10(b)]. Moreover, asymmetries in the charges of the water islands or in the particle
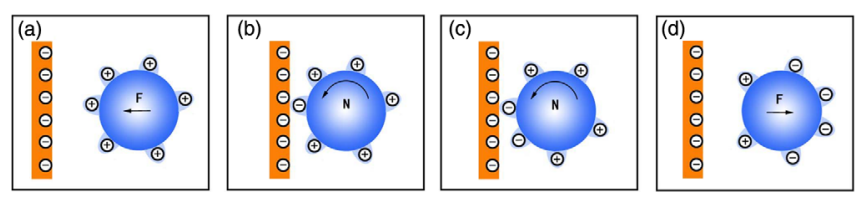

FIG. 10. (a)-(d) Charging mechanism of a hydrophobic particle in contact with the cathode. shape induce torsion, and the particle thus rolls under its action [see Fig. 10(c)]. During this rolling process, more and more isolated adsorbed water domains become equipotential with the cathode, and the particles thus acquire more negative charges [see Fig. 10(c)]. Once the net charge of the insulator becomes negative, the particle separates from the cathode [see Fig. 10(d)].

[1] T. Shinbrot and H. J. Herrmann, Granular Matter: Static in Motion, Nature (London) 451, 773 (2008).

[2] D. J. Lacks, Particle Clouds Frictile Attraction, Nat. Phys. 6, 324 (2010).

[3] J. F. Kok and N. O. Renno, Electrostatics in Wind-Blown Sand, Phys. Rev. Lett. 100, 014501 (2008).

[4] J.P. Merrison, Sand Transport, Erosion and Granular Electrification, Aeolian. Res. 4, 1 (2012).

[5] S. R. McNutt and C. M. Davis, Lightning Associated with the 1992 Eruptions of Crater Peak, Mount Spurr Volcano, Alaska, J. Volcanol. Geotherm. Res. 102, 45 (2000).

[6] T. Abbasi and S. A. Abbasi, Dust Explosions-Cases, Causes, Consequences, and Control, J. Hazard. Mater. 140, 7 (2007).

[7] L. Schein, Comparison of Toner Adhesion Theories, J. Imaging Sci. Technol. 53, 010506 (2009).

[8] E. S. Polsen, A. G. Stevens, and A. J. Hart, Laser Printing of Nanoparticle Toner Enables Digital Control of Micropatterned Carbon Nanotube Growth, ACS Appl. Mater. Interfaces 5, 3656 (2013).

[9] H. Kawamoto, in Proceedings of International Conference on Digital Printing Technologies and Digital Fabrication (Society for Imaging Science and Technology, Anchorage, 2007), Vol. 23, p. 961.

[10] P. Calvert, Inkjet Printing for Materials and Devices, Chem. Mater. 13, 3299 (2001).

[11] B. A. Kwetkus, Particle Triboelectrification and Its Use in the Electrostatic Separation Process, Part. Sci. Technol. 16, 55 (1998).

[12] G. S. P. Castle, in Proceedings of the ESA Annual Meeting on Electrostatics 2008 (Electrostatics Society of America, Minneapolis, 2008), p. M1.

[13] L. S. McCarty and G. M. Whitesides, Electrostatic Charging Due to Separation of Ions at Interfaces: Contact Electrification of Ionic Electrets, Angew. Chem., Int. Ed. Engl. 47, 2188 (2008).

[14] D. J. Lacks and R. M. Sankaran, Contact Electrification of Insulating Materials, J. Phys. D 44, 453001 (2011).

[15] T. Pähtz, H. J. Herrmann, and T. Shinbrot, Why Do Particle Clouds Generate Electric Charges?, Nat. Phys. 6, 364 (2010).

[16] L. B. Schein, Recent Progress and Continuing Puzzles in Electrostatics, Science 316, 1572 (2007).

[17] C. Liu and A. J. Bard, Electrostatic Electrochemistry at Insulators, Nat. Mater. 7, 505 (2008).

[18] A. F. Diaz and R. M. Felix-Navarro, A Semi-quantitative Tribo-electric Series for Polymeric Materials: The Influence of Chemical Structure and Properties, J. Electrost. 62, 277 (2004). 
[19] H. T. Baytekin, A. Z. Patashinski, M. Branicki, B. Baytekin, S. Soh, and B. A. Grzybowski, The Mosaic of Surface Charge in Contact Electrification, Science 333, 308 (2011).

[20] Z. Gu, W. Wei, J. Su, and C. W. Yu, The Role of Water Content in Triboelectric Charging of Wind-Blown Sand, Sci. Rep. 3, 1337 (2013).

[21] T. R. Ducati, L. H. Simões, and F. Galembeck, Charge Partitioning at Gas-Solid Interfaces: Humidity Causes Electricity Buildup on Metals, Langmuir 26, 13763 (2010).

[22] M. W. Williams, What Creates Static Electricity?, Am. Sci. 100, 316 (2012).

[23] T. A. L. Burgo, C. A. Silva, L. B. S. Balestrin, and F. Galembeck, Friction Coefficient Dependence on Electrostatic Tribocharging, Sci. Rep. 3, 2384 (2013).

[24] M. W. Williams, Triboelectric Charging of Insulators-Mass Transfer versus Electrons/Ions, J. Electrost. 70, 233 (2012).

[25] N. Knorr, Squeezing Out Hydrated Protons: Low-FrictionalEnergy Triboelectric Insulator Charging on a Microscopic Scale, AIP Adv. 1, 022119 (2011).

[26] A. K. Kamra, Visual Observation of Electric Sparks on Gypsum Dunes, Nature (London) 240, 143 (1972).

[27] T. A. L. Burgo, C. A. Rezende, S. Bertazzo, A. Galembeck, and F. Galembeck, Electric Potential Decay on Polyethylene: Role of Atmospheric Water on Electric Charge Buildup and Dissipation, J. Electrost. 69, 401 (2011).

[28] J. A. Wiles, M. Fialkowski, M. R. Radowski, G. M. Whitesides, and B.A. Grzybowski, Effects of Surface Modification and Moisture on the Rates of Charge Transfer between Metals and Organic Materials, J. Phys. Chem. B 108, 20296 (2004).

[29] R. F. Gouveia and F. Galembeck, Electrostatic Charging of Hydrophilic Particles Due to Water Adsorption, J. Am. Chem. Soc. 131, 11381 (2009).

[30] S. Pence, V. J. Novotny, and A. F. Diaz, Effect of Surface Moisture on Contact Charge of Polymers Containing Ions, Langmuir 10, 592 (1994).

[31] H. T. Baytekin, B. Baytekin, S. Soh, and B. A. Grzybowski, Is Water Necessary for Contact Electrification?, Angew. Chem. 50, 6766 (2011).

[32] X. Zheng, R. Zhang, and H. Huang, Theoretical Modeling of Relative Humidity on Contact Electrification of Sand Particles, Sci. Rep. 4, 4399 (2014).

[33] J. F. Kok and N. O. Renno, Enhancement of the Emission of Mineral Dust Aerosols by Electric Forces, Geophys. Res. Lett. 33, L19S10 (2006).

[34] M. Sow, A. R. Akande, K. S. Robinson, R. M. Sankaran, and D. J. Lacks, The Role of Humidity on the Lift-Off of
Particles in Electric Fields, J. Braz. Chem. Soc. 24, 273 (2013).

[35] N. N. Levedev and I. P. Skalskaya, Force Acting on a Conducting Sphere in the Field of a Parallel Plate Condenser, Sov. Phys. Tech. Phys. Engl. Transl. 7, 268 (1962).

[36] K. Xu, P. Cao, and J. R. Health, Graphene Visualizes the First Water Adlayers on Mica at Ambient Conditions, Science 329, 1188 (2010).

[37] J. Carrasco, A. Hodgson, and A. Michaelides, A Molecular Perspective of Water at Metal Interfaces, Nat. Mater. 11, 667 (2012).

[38] See Supplemental Material at http://link.aps.org/ supplemental/10.1103/PhysRevX.5.011002 for three movies showing the bouncing of different objects between the electrodes.

[39] W. D. Ristenpart, J. C. Bird, A. Belmonte, F. Dollar, and H. A. Stone, Non-coalescence of Oppositely Charged Drops, Nature (London) 461, 377 (2009).

[40] V. Chakrapani, J. C. Angus, A. B. Anderson, S. D. Wolter, B. R. Stoner, and G. U. Sumanasekera, Charge Transfer Equilibria between Diamond and an Aqueous Oxygen Electrochemical Redox Couple, Science 318, 1424 (2007).

[41] G. E. Ewing, Ambient Thin Film Water on Insulator Surfaces, Chem. Rev. 106, 1511 (2006).

[42] T. Siu, J. Cotton, G. Mattson, and T. Shinbrot, SelfSustaining Charging of Identical Colliding Particles, Phys. Rev. E 89, 052208 (2014).

[43] C. A. Rezende, R. F. Gouveia, M. A. da Silva, and F. Galembeck, Detection of Charge Distributions in Insulator Surfaces, J. Phys. Condens. Matter 21, 263002 (2009).

[44] D. J. Im, M. M. Ahn, B. S. Yoo, D. Moon, D. W. Lee, and I. S. Kang, Discrete Electrostatic Charge Transfer by the Electrophoresis of a Charged Droplet in a Dielectric Liquid, Langmuir 28, 11656 (2012).

[45] D. J. Im, J. Noh, D. Moon, and I. S. Kang, Electrophoresis of a Charged Droplet in a Dielectric Liquid for Droplet Actuation, Anal. Chem. 83, 5168 (2011).

[46] O. Enkgvist and A. J. Stone, Intermolecular Potentials and Low Temperature Structures, J. Chem. Phys. 110, 12089 (1999).

[47] D. B. Asay and S. H. Kim, Evolution of the Adsorbed Water Layer Structure on Silicon Oxide at Room Temperature, J. Phys. Chem. B 109, 16760 (2005).

[48] L. P. Santos, R. T. R. D. Ducati, L. B. S. Balestrin, and F. Galembeck, Water with Excess Electric Charge, J. Phys. Chem. C 115, 11226 (2011). 